\title{
Complete Cure in COVID-19 Patient with Respiratory and Gastrointestinal Symptoms
}

\author{
Ghada Mahmoud Abdel-Rafee
}

Department of Radiodiagnosis, Mansoura Health Insurance Hospital, Mansoura, Egypt.

Correspondence to: Ghada Mahmoud Abdel-Rafee, department of radiodiagnosis, Mansoura Health Insurance Hospital, Mansoura Egypt

Email:

doctorghada2016@yahoo.com

Received: 6 May 2020

Accepted: 17 May 2020

\section{Abstract:}

Background: COVID-19 is fatal disease caused by SARS-CoV-2. It spread more rapidly than SARS, MERS \& common cold due to increase globalization \& adaptation of the virus in every environment. SARSCOV-2 is the seventh known coronavirus which includes sever acute respiratory syndrome (SARS) \& Middle East Respiratory Syndrome (MERS). The structure of the virus is behind its varying symptoms \& the defense mechanism. It is important to consider respiratory, gastrointestinal \& neurological symptoms. Also travel history \& close contact of positive COVID-19 cases as well. Prompt recognition of imaging patterns based on the infection time course is essential for not only understanding the natural history of infection, but also for helping to predict patient evolution \& possible complication development. Subject and method: We report a female patient case of COVID-19 with respiratory \& gastrointestinal manifestations, imaging findings \& detection of response to the treatment regimen. Result: Marvelous response of the treatment regimen $\&$ complete cure of the respiratory \& gastrointestinal symptoms. Conclusion: The course of coronavirus 2019 disease can't be predicted. The discussed treatment protocol is an evolution in the age of COVID-19.

Keywords: COVID-19, SARS-CoV-2, MERS.

\section{Introduction:}

Coronavirus disease-19 (COVID-19) is a disease caused by sever acute respiratory syndrome-coronavirus-2 (SARS-VOV-2). It was initially reported in Wuhan, China in
December, 2019 \& is currently spreading world-wide (1). The World Health Organization (WHO) started that COVID-19 was a global health emergency on 30 
January, 2020 \& classified it as a pandemic on March, 2020.

During the early course of infection, main lung abnormalities include peripheral focal or multifocal ground glass opacities affecting both lungs in approximately 50\%$75 \%$ of patients. As the progression of the disease, crazy paving \& consolidation become the major CT findings, peaking around 9-13 days of the disease. Then, it's usually followed by a gradual cleaving at near 1 month \& beyond (2). Pleural effusions \& mediastinal lymphadenopathy are usually absent in COVID-19 patients. Finally, it is important to point out that chest $\mathrm{CT}$ is more sensitive than chest $\mathrm{x}$-ray in detection of ground glass opacities (3).

\section{Subject \& method:}

A case report study, that was conducted in Mansoura Health Insurance Hospital in 1 to 7 May, 2020 time range. Written patient consent for publication was taken. We report a case of 58 years old female patient, presented with fever (38 C), dry cough \& generalized fatigue for 3 days. She denied dyspnea \& chest pain. She had nausea, vomiting, diarrhea $\&$ epigastric pain for 2 days. Laboratory studies revealed elevated serum ferritin $(244.70 \mathrm{ng} / \mathrm{ml}$, normal range is $13-159 \mathrm{ng} / \mathrm{ml})$, elevated serum LDH (299 $\mathrm{U} / \mathrm{L}$, normal range is $135-214 \mathrm{U} / \mathrm{L}$ ), elevated serum C-Reactive protein $(23.69 \mathrm{mg} / \mathrm{L}$, normal range is $0-5 \mathrm{mg} / \mathrm{dl}$ ) \& decreased total leucocytic count $(2.9 \times 1000$ cells $/ \mathrm{mm} 3$, normal range is $4.3-10.8 \mathrm{gm} / \mathrm{dl}$ ) with obvious neutropenia \& lymphopenia. The patient was hospitalized for further care.

\section{Results:}

In our case, plain $\mathrm{x}$-ray chest (PA view) revealed accentuated bronchovascular marking on both sides \& minimal reticulonodular opacity in both lung bases (Fig. 1). NCCT chest demonstrated multifocal bilateral patchy ground glass opacities with a predominantly lower lung zones \& peripheral distribution. No pleural effusion or pulmonary cavitation was found (Fig.2,3). The treatment protocol includes Ostelamivir tab, Averzolid tab, Amrizol (500mg) tab, Abimol tab, Xithrone (500mg) tab \& Asthmalcat $(10 \mathrm{mg})$ tab for 1 week \& re-check of symptoms revealed complete cure of fever, cough \& muscle pain. Laboratory investigation revealed improved serum $\mathrm{C}$ - reactive protein $(9.92 \mathrm{mg} / \mathrm{L}) \&$ improved total leucocytic count (3.3 X 1000 cells/mm3) with relative lymphocytosis \& absolute neutropenia. Non-contrast CT chest revealed complete cure \& clear both lung 
fields which plays a crucial diagnostic tool (Fig. 4).

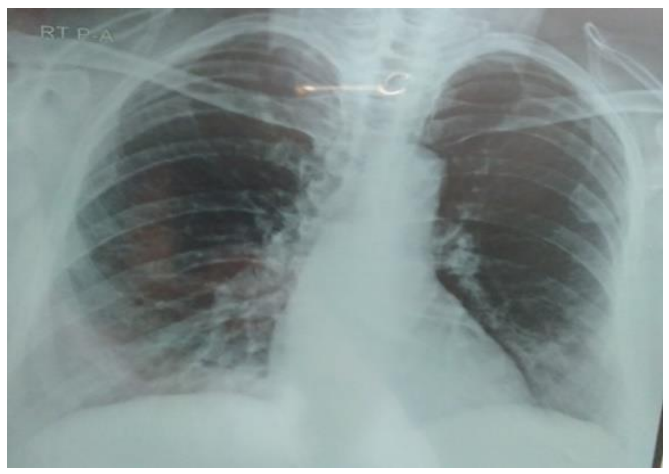

Figure (1): Plain x-ray chest (PA view), revealed accentuated bronchovascular marking on both sides \& minimal reticulonodular opacity in both lung bases.

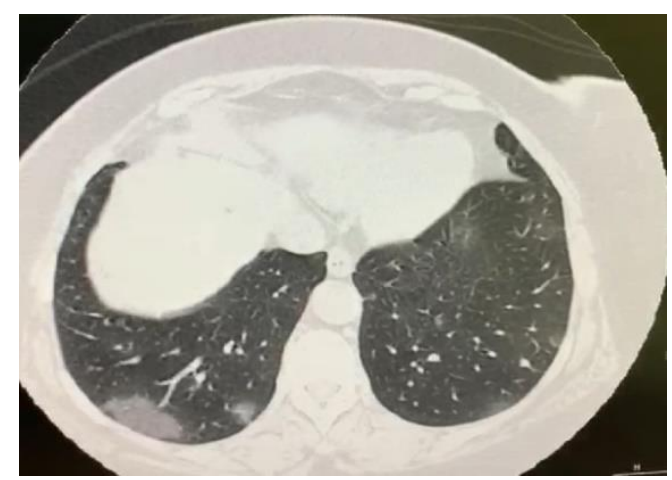

Figure (2): NCCT chest (axial cut), revealed typical findings of COVID-19 as: peripheral bilateral multilobar ground glass opacities \& fine septal thickening.

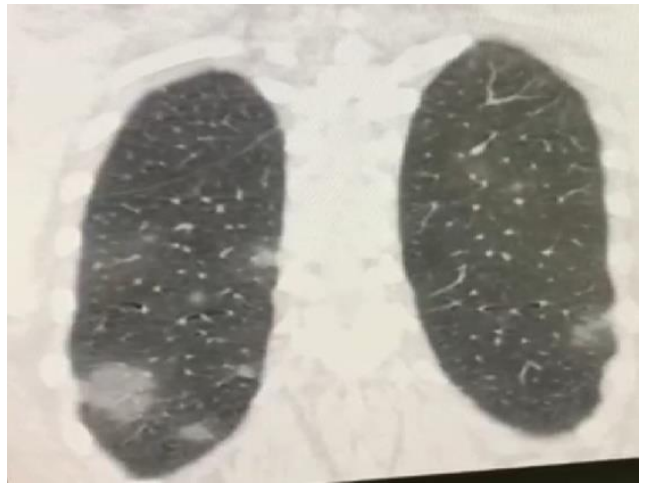

Figure (3): NCCT chest (coronal cut), revealed peripheral bilateral sub-pleural rounded ground glass opacities, more in lower lung zone.

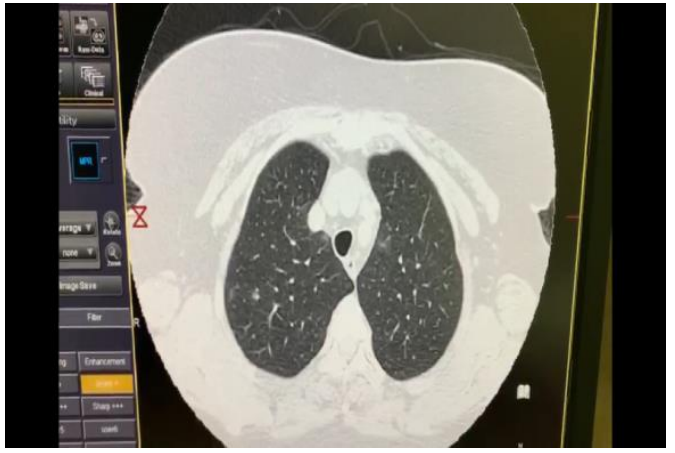

Figure (4): NCCT chest after 1 week of treatment (axial cut), revealed complete cure \& clear both lung fields

\section{Discussion}

The majority of patients with lower respiratory tract infections caused by COVID-19 presents with cough, fever \& other non-specific symptoms including dyspnea, myalgia, headache \& fatigue. (4) About $20 \%$ of cases are severed \& fatality rate is approximately $3 \%$. (5) Some patients may also present early gastrointestinal symptoms including vomiting, nausea \& diarrhea, which preceded respiratory symptoms (6). Real-time polymerase chain reaction (RT-PCR) is the current standard diagnostic method used to detect viral nucleotides from specimens obtained by nasopharyngeal swap, oropharyngeal swab, bronchoalveolar lavage or tracheal aspirate. Nevertheless, this method sensitivity is as low as $60 \%-70 \%$ for detecting SARS-COV2, which can probably be attributed to the inadequate sample volume, contaminated samples \& collection in an inappropriate 

time window. By contrast, CT chest has revealed about 56\%-98\% sensitivity in detecting COVID-19 even at initial presentation \& can be useful in correcting false negatives acquired from RT-PCR during initial stages of infection (7). Thoracic imaging has a crucial role in the evaluation of patients suspected of COVID-19. The number of infected individual is constantly on the rise $\&$ the treatment protocol is symptom based mainly \& the seriously ill individuals are provided with organ support. The major pipeline drugs are antiviral drugs, Remidesivir, Choloroquine, Favipiravir \& lopinavir. (8) By the way general precautions must be considered as hand washing by $70 \%$ alcohol, vitamin $\mathrm{C}$ supplement, avoids face contact by hands \& keeps social distancing.

\section{Conclusion:}

It is essential to conduct integrated approach of imaging \& molecular diagnosis would help in screening \& treating COVID-19 effectively.

\section{References:}

1. Kaune J, Little B \& Chung J. (2020): Essentials for Radiologists on COVID-19: An Update-Radiology
Scientific Expert Panel. Radiology. (PMID: 32105562).

2. Bernheim A, Mei X. \& Huang M. (2020): Chest CT findings in coronavirus disease-19 (COVID-19). Relationship to duration of infection. Radiology. (PMID: 32077789).

3. Ming-Yen NG, Elaine YPL \& Jin Y. (2020): Imaging profile of the COVID-19 infection: Radiologic findings \& literature Review. Radiology.

4. Huang C, Wang Y \& Li X. (2020): Clinical features of patients infected with 2019 novel coronavirus in Wuhan, China. Lancet.

5. Wu Z. \& Mc Googan JM. (2020): Characteristics of important lessons from the coronavirus disease 2019 (COVID-19) outbreak in China: Summary of a report of a 72314 cases from the Chinese Center for Disease Control \& Prevention. JAMA.

6. Xiao F., Tang M. \& Zheng X. (2020): Evidence of gastrointestinal infection of SARS-COV-2. Gastroenterology. (PMID: 32142773).

7. Bai H., Hseih B. \& Yiong Z. (2020): Performance of Radiologists in differentiating COVID-19 from viral pneumonia on chest CT. Radiology. (PMID: $32155105)$.

8. Jin Y.H., Cai L, Cheng Z.S., Cheng H., Deng T., Fan Y.P. et al. (2020): A rapid advice guideline for the diagnosis \& treatment of 2019 novel coronavirus (2019-nCov) infected pneumonia (standard version). Military. Med. Res.7,4. (https://doi.org/10.1186/s40779-020-0233-6).

To cite this article: Ghada Mahmoud Abdel-Rafee. Complete Cure in COVID-19 Patient with Respiratory and Gastrointestinal Symptoms. BMFJ 2020;37(Radiology):17-20, DOI: 10.21608/bmfj.2020.29622.1258 